\title{
Aspirin protects human coronary artery endothelial cells by inducing autophagy
}

\author{
J. CHEN ${ }^{\dagger}$, L. WANG ${ }^{\dagger}$, W.H. LIU, J. SHI, Y. ZHONG, S.J. LIU and S.M. LIU*
}

Guangzhou Institute of Cardiovascular Disease, Guangdong Key Laboratory of Vascular Diseases, State Key Laboratory of Respiratory Disease, The Second Affiliated Hospital of Guangzhou Medical University, Guangzhou, Guangdong 510260, PR China

Received: November 17, 2019 • Accepted: May 13, 2020

Published online: July 24, 2020

(C) 2020 Akadémiai Kiadó, Budapest

\section{ABSTRACT}

Although the use of aspirin has substantially reduced the risks of cardiovascular events and death, its potential mechanisms have not been fully elucidated. In a previous study, we found that aspirin triggers cellular autophagy. In the present study, we aimed to determine the protective effects of aspirin on human coronary artery endothelial cells (HCAECs) and explore its underlying mechanisms. HCAECs were treated with oxidized low-density lipoprotein (ox-LDL), angiotensin II (Ang-II), or high glucose (HG) with or without aspirin stimulation. The expression levels of endothelial nitric oxide (NO) synthase (eNOS), p-eNOS, LC3, p62, phosphor-nuclear factor kappa B (p-NF- $\kappa$ B), p-p38 mitogen-activated protein kinase (p-p38 MAPK), and Beclin-1 were detected via immunoblotting analysis. Concentrations of soluble intercellular adhesion molecule-1 (sICAM-1) and soluble vascular cell adhesion molecule-1 (sVCAM-1) were measured via ELISA. NO levels were determined using the Griess reagent. Autophagic flux was tracked by tandem mRFP-GFP-tagged LC3. Results showed that aspirin increased eNOS level and reduced injury to the endothelial cells (ECs) caused by ox-LDL, Ang-II, and HG treatment in a dose-dependent manner. Aspirin also increased the LC3II/LC3I ratio, decreased p62 expression, and enhanced autophagic flux (autophagosome and autolysosome puncta) in the HCAECs. p-NF- $\kappa \mathrm{B}$ and p-p38 mitogen-activated protein kinase inhibition, sVCAM-1 and sICAM-1 secretion, and eNOS activity promotion by aspirin treatment were found to be dependent on Beclin-1. These results suggested that aspirin can protect ECs from ox-LDL-, Ang-II-, and HG-induced injury by activating autophagy in a Beclin-1-dependent manner.

\footnotetext{
*Corresponding author. Tel.: +86 20 34153256, E-mail: liushiming@gzhmu.edu.cn
}

\footnotetext{
${ }^{\dagger}$ Jing Chen and Li Wang contributed equally to this work.
} 


\section{KEYWORDS}

aspirin, endothelial cells, autophagy, Beclin-1, protection

\section{INTRODUCTION}

Atherosclerosis is the main pathology of coronary artery diseases. Endothelial cell (EC) dysfunction is the initial stage of atherosclerosis. EC functions play a vital role in maintaining vascular homeostasis, blood flow and pressure control, and balancing insurance of anticoagulant and antithrombotic properties [1]. Protection and enhancement of EC functions are beneficial for the prevention and treatment of coronary artery diseases. Oxidized low-density lipoprotein (ox-LDL) has a vital role in atherosclerosis development [2]. ox-LDL decreases EC proliferation, migration, and tube formation, which are processes that might be associated with nuclear factor kappa B (NF- $\kappa \mathrm{B})$ p65 activation and vascular endothelial growth factor downregulation [3]. oxLDL also promotes foam cell formation, which exerts a chemical toxicity effect on smooth muscle cells to accelerate smooth muscle cell migration. Angiotensin II (Ang-II) induces inflammatory responses and leads to hypertension and coronary artery diseases by activating NF$\kappa \mathrm{B}$ [4]. High glucose (HG) also induces endothelial dysfunction, but the underlying mechanism has not been fully elucidated. Production of different cytokines in ECs has been found impaired under HG conditions [5].

Aspirin, which is widely used in medicine, remarkably reduces the risks of cardiovascular events and death. Aspirin has excellent antiplatelet functions and powerful vascular endothelial protection effects [6-10]. The mechanisms by which aspirin protects EC functions have been investigated. Aspirin can promote nitric oxide (NO) levels by directly stimulating the endothelial NO synthase (eNOS) [11]. Aspirin can exert protective effects on ox-LDL-induced endothelial injury by inhibiting cyclooxygenase- 2 and intercellular adhesion molecule-1 (ICAM-1) expression [12]. Aspirin also protects against Ang-II-induced end organ damage by inhibiting NF- $\kappa \mathrm{B}$ [4]. Furthermore, aspirin alters HG-induced changes in cellular $\mathrm{Ca}^{2+}$ homeostasis and $\mathrm{NO}$ production involving the reduction of $\mathrm{O}^{2-}$ levels [11].

Autophagy is a life phenomenon in eukaryotic cells that plays a valuable role in metabolism. The LC3 II/I ratio is used as an indicator of autophagy-mitophagy flux. The p62 protein, which is also called SQSTM1, SQSTM1/p62 binds the autophagosome membrane protein LC3/Atg8, thereby transporting a protein polymer containing SQSTM1/p62 to the autophagosome. In autophagy, autophagosome degradation by lysosomes decreases SQSTM1/p62 levels. Mammalian Beclin-1, which is an ortholog of the Atg6/vacuolar protein sorting (Vps)-30 protein in yeast, participates in the autophagic process. The Beclin-1-Vps34 complex facilitates lipid membrane extension, cargo recruitment, and autophagosome maturation [13, 14]. Autophagosomes and autophagic lysosome formation are markers of autophagy in cells [15]. Available evidence suggests that aspirin works effectively in the primary prevention of various tumors, such as colon, breast, lung, and prostate tumors [16], and its mechanisms have generated interest among researchers. Aspirin features an autophagic effect on colorectal cancer 
cells by inhibiting the mammalian target of rapamycin (mTOR) signaling and activating the adenosine 5 -monophosphate-activated protein (AMPK) pathway [17]. In a previous study, we found that aspirin reduces the incidence of hepatocellular carcinoma (HCC) and plays a synergistic role with chemotherapy for HCC treatment that might be related to induced autophagy [18].

However, the relationship between autophagy induction and vascular endothelial protection by aspirin has not been elucidated yet [6-10]. In the present study, we aimed to determine whether aspirin can protect human coronary artery ECs (HCAECs) by inducing autophagy under different injury conditions (ox-LDL, Ang-II, and HG) and explore its underlying mechanisms.

\section{MATERIALS AND METHODS}

\section{Cell culture and treatment}

HCAECs were purchased from Cell Applications, Inc. (San Diego, CA, USA). The HCAECs were maintained at $37{ }^{\circ} \mathrm{C}$ in a humidified mixture of $95 \%$ air and $5 \% \mathrm{CO}_{2}$ in EBM-2 medium (Cat. No. CC3162; Lonza, USA) with endothelial growth medium supplement mix (hFGF, VEGF, IGF-1, hEGF, hydrocortisone, heparin, and GA-1000) supplemented with 5\% fetal bovine serum and $1 \%$ penicillin-streptomycin (Gibco, USA). Cells between four and six passages were used in all experiments. The cells were treated with or without aspirin in a dosedependent manner $(0.315,0.63,1.25,2.5$, and $5 \mathrm{mM}$ ) (Sangon Biotech, Shanghai, China) for 16 h. Alternatively, the cells were stimulated using $50 \mu \mathrm{g} / \mathrm{mL}$ ox-LDL (Yiyuan Biotechnology, China), $1 \mu \mathrm{M}$ Ang-II (Sigma-Aldrich, USA), and $25 \mathrm{mM} \mathrm{HG}$ (Sigma-Aldrich, USA) for $30 \mathrm{~min}$, and then aspirin $(2.5 \mathrm{mM})$ was used to stimulate the cells for $12 \mathrm{~h}$.

\section{Immunoblotting analysis}

HCAECs were grown in six-well plates at approximately $60-70 \%$ confluence for $24 \mathrm{~h}$ and then treated with aspirin at indicated concentrations or times. The cells were harvested and subjected to immunoblotting analysis. Immunoblotting was performed in accordance with our previously described method [18]. In brief, equal amounts of total protein were separated using $8 \%$ or $12 \%$ SDS-PAGE and transferred to PVDF membranes (Millipore, USA). The membranes were blocked in 5\% nonfat milk (Bio-Rad Laboratories, USA) for $1 \mathrm{~h}$ and then incubated with primary antibodies overnight at $4{ }^{\circ} \mathrm{C}$. Goat anti-rabbit or anti-mouse IgG conjugated with HRP (1:5,000; Jackson ImmunoResearch, USA) served as secondary antibodies. Finally, immunoreactive bands were detected via the exposure method. The antibodies used in this study were as follows: LC3B ( $1 \mu \mathrm{g} / \mathrm{mL}$, \#L7543; Sigma-Aldrich, USA), p-eNOS (1:1,000, \#9571s; Cell Signaling Technology, USA), eNOS (1:1,000, \#9572s; Cell Signaling Technology, USA), GAPDH (1:2,000, \#2118L; Cell Signaling Technology, USA), phosphor-NF- $\kappa$ B (p-NF- $\kappa \mathrm{B}, 1: 1,000$, \#3033s; Cell Signaling Technology, USA), p62 (1:1,000, \#5114; Cell Signaling Technology, USA), Beclin-1 (1:1,000, \#3495s; Cell Signaling Technology, USA), and p-p38 mitogen-activated protein kinase (1:1,000, \#4511s; Cell Signaling Technology, USA). 


\section{NO measurement}

NO levels were detected by Griess reaction on the basis of the total concentrations of NO stable end products, namely, nitrite and nitrate. Following the manufacturer's instructions of a commercial NO assay kit (Beyotime Institute of Biotechnology, Shanghai, China), the culture medium from cultures subjected to different treatments was collected, and $50 \mu \mathrm{L}$ of culture medium, $50 \mu \mathrm{L}$ of Griess reagent I, and $50 \mu \mathrm{L}$ of Griess reagent II were added to each well of 96well plates at room temperature for $2 \mathrm{~min}$, as previously described [19, 20] (PMID: 28413519). Then, the optical density (OD) of the 96-well plates was measured at $540 \mathrm{~nm}$ on a plate reader (Epoch Microplate Spectrophotometer, Bio Tek, USA). NO production was then calculated by comparing the $\mathrm{OD}$ values of the samples with the standard curve.

\section{ELISA assay}

Soluble intercellular adhesion molecule-1 (sICAM-1) and soluble vascular cell adhesion molecule-1 (sVCAM-1) levels in the culture media after different treatments were measured using commercially available ELISA kits (Dakewei Biotech, Shenzhen, China). Following the manufacturer's instructions, the culture media were centrifuged at $2,000 \mathrm{rpm}$ for $15 \mathrm{~min}$ at $4{ }^{\circ} \mathrm{C}$ to remove cell debris. The plates were mixed with biotinylated antibody working solution (1:50, 50 $\mu \mathrm{L} /$ well) specific for sICAM-1 for $1 \mathrm{~h}$. The plates were incubated with biotinylated antibody working solution (1:100, $100 \mu \mathrm{L} /$ well $)$ specific for sVCAM-1 for $2 \mathrm{~h}$ after they were incubated for $2 \mathrm{~h}$ with the standards and samples and washed. The plates washed with $1 \times$ washing buffer (three times, $1 \mathrm{~min} /$ time) were incubated with streptavidin-HRP working solution $(100 \mu \mathrm{L} /$ well $)$ for $20 \mathrm{~min}$. Subsequently, the plates were washed with $1 \times$ washing buffer (three times, $1 \mathrm{~min} /$ time) and added with $100 \mu \mathrm{L} /$ well TMB substrate solution in the dark for 10-15 min. The enzyme-substrate reaction was stopped by quickly pipetting $100 \mu \mathrm{L}$ of the stop solution into each well. OD values were measured at $450 \mathrm{~nm}$ on a plate reader, and the results were calculated by comparing the standards with the blanks. The medium concentration was calculated as per microgram of cell protein in each well.

\section{Monitoring autophagic flux using tandem mRFP-GFP-tagged LC3}

HCAECs were grown in 12-well plates to approximately $80 \%$ confluence and then infected with adenovirus harboring tandem fluorescent mRFP-GFP-LC3 (HanBio, Shanghai, China) for $24 \mathrm{~h}$. The cells were treated with or without $2.5 \mathrm{mM}$ aspirin or $100 \mathrm{nM}$ rapamycin (Sangon Biotech, Shanghai, China) for $16 \mathrm{~h}$, and the green and red fluorescence was analyzed via fluorescence microscopy. The number of autophagosomes (dots in yellow in merged images) and autolysosomes (dots in red only and not in green) in images was visually counted in five randomly selected fields per group. Images were analyzed using Image J.

\section{Small interfering RNA}

Beclin-1 small interfering RNA (siRNA) and negative control (NC) siRNA were synthesized by GenePharma (Suzhou, China), the sequences of which were as follows: 


\begin{tabular}{lc}
\hline NC siRNA & Forward: 5'-UUCUCCGAACGUGUCACGUTT-3' \\
& Reverse: 5'-ACGUGACACGUUCGGAGAATT-3' \\
Beclin-1 siRNA \#1 & Forward: 5' 5.UCGCUGAAGACAGAGCGAUTT-3' $^{\prime}$ \\
& Reverse: 5'-AUCGCUCUGUCUUCAGCGATT-3' \\
Beclin-1 siRNA \#2 & Forward: 5'-AGUUGGAUAAUGUGGGAAATT-3' \\
& Reverse: 5'-UUUCCCACAUUAUCCAACUTT-3' \\
Beclin-1 siRNA \#3 & Forward: 5'-GAGACAUUAUGGAGAGAUUTT-3' \\
& Reverse: 5'-AAUCUCUCCAUAAUGUCUCTT-3' \\
Beclin-1 siRNA \#4 & Forward: 5'-CAGUUUGGCACAAUCAAUATT-3', \\
& Reverse: 5'-UAUUGAUUGUGCCAAACUGTT-3'
\end{tabular}

HCAECs were seeded in six-well plates and grown to $70-80 \%$ confluence. The cells were transfected with NC siRNA (50 nM) or Beclin-1 siRNA (50 nM) by using Lipofectamine 3000 (Thermo Fisher Scientific, Inc., USA) according to the manufacturer's instructions. After $48 \mathrm{~h}$ of transfection, the cells were treated with or without $2.5 \mathrm{mM}$ aspirin for $16 \mathrm{~h}$.

\section{Statistical analysis}

Statistical analysis was performed using SPSS 16.0 software (SPSS, Inc., USA) and GraphPad Prism 6 software (GraphPad Software, USA). Data from at least three independent experiments were presented as the mean \pm standard deviation. Different treatment groups were compared using Student's $t$-test or ANOVA when appropriate. Statistical significance was considered at $P<0.05$.

\section{RESULTS}

\section{Aspirin increased eNOS activity in a dose-dependent manner}

To determine the effects of aspirin on HCAEC functions, we detected the levels of p-eNOS protein expression and NO. Results showed that p-eNOS (Fig. 1A) and NO levels (Fig. 1B) increased in a dose-dependent manner under aspirin treatment compared with control values, indicating that aspirin enhanced eNOS activity in ECs.

\section{Aspirin protected ECs against ox-LDL, Ang-II, and HG injuries}

To determine whether aspirin reduces EC damage caused by ox-LDL, Ang-II, and HG, we detected the expression levels of p-p38, p-NF- $\kappa$ B, sICAM-1, and sVCAM-1. Results showed that $50 \mu \mathrm{g} / \mathrm{mL}$ ox-LDL, $1 \mu \mathrm{M}$ Ang-II, and $25 \mathrm{mM}$ HG substantially increased p-p38 and p-NF- $\kappa \mathrm{B}$ protein expression levels in the HCAECs, and aspirin reduced p-p38 and p-NF- $\kappa$ B protein expression levels (Fig. 2A). ox-LDL, Ang-II, and HG also remarkably increased sICAM-1 and sVCAM-1 expression levels in the HCAECs, but aspirin reduced sICAM-1 and sVCAM-1 expression levels (Fig. 2B). 


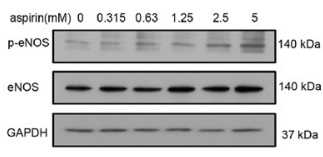

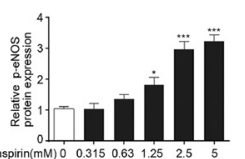

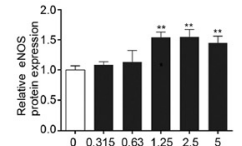

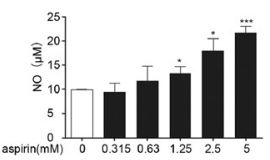

Fig. 1. Aspirin increased p-eNOS level and NO concentration in the HCAECs. HCAECs were incubated with aspirin at various concentrations $(0,0.315,0.63,1.25,2.5$, and $5 \mathrm{mM})$ for $16 \mathrm{~h}$. Cell lysates were subjected to immunoblotting assay with antibodies against p-eNOS, eNOS, and GAPDH. (A) The left panel shows the representative data. The right panel presents the band quantification of three experiments. (B) Cell lysates were subjected to Griess reagent to determine NO levels. Each bar represents the mean \pm SD calculated from three independent experiments; ${ }^{\star} P<0.05$, ${ }^{* \star} P<0.01$, and ${ }^{\star *} P<0.001$ : aspirin treatment was compared with the control

\section{Aspirin triggered EC autophagy}

We explored the underlying mechanisms by which aspirin protects vascular ECs from damage induced by ox-LDL, Ang-II, and HG. Results showed that 0.63, 1.25, and $2.5 \mathrm{mM}$ aspirin increased the LC3II/LC3I ratio but reduced p62 expression in the HCAECs in a dose-dependent

A
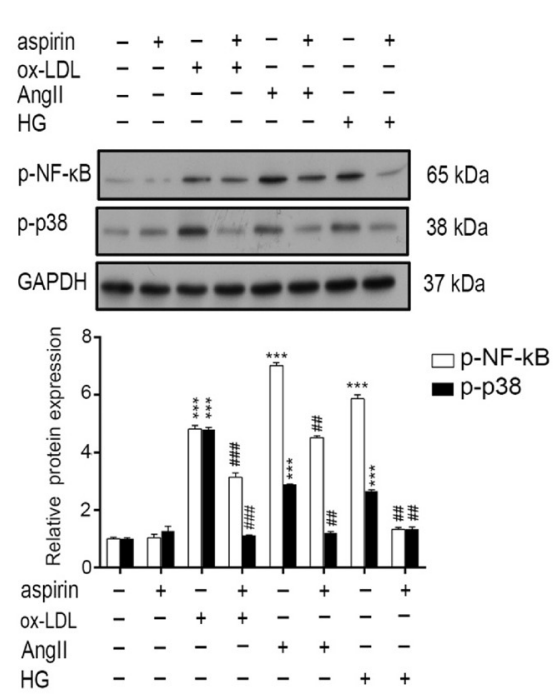

B

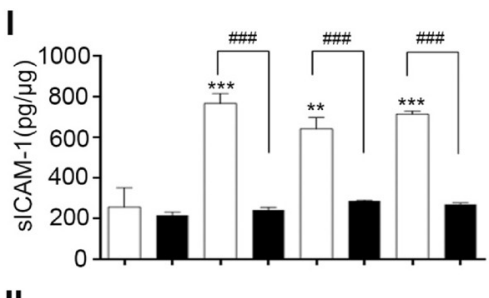

II

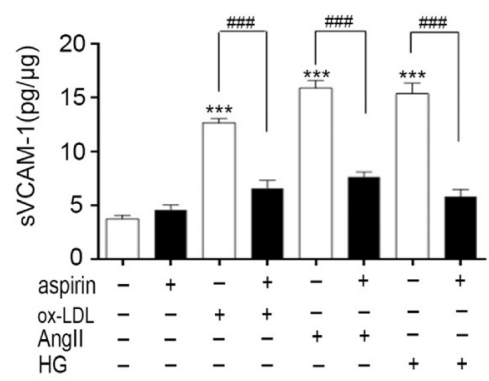

Fig. 2. Aspirin reduced injury to HCAECs caused by ox-LDL, Ang-II, and HG. (A) HCAECs were incubated with $2.5 \mathrm{mM}$ aspirin, $50 \mu \mathrm{g} / \mathrm{mL}$ ox-LDL, $1 \mu \mathrm{M}$ Ang-II, and $25 \mathrm{mM} \mathrm{HG}$ for $12 \mathrm{~h}$. Cell lysates were subjected to immunoblotting assay with antibodies against p-P38, p-NF- $\kappa \mathrm{B}$, and GAPDH. The upper panel shows the representative data. The lower panel presents the band quantification of three experiments. (B) ELISA was performed to determine (I) sICAM-1 and (II) sVCAM-1 expression levels in the HCAEC culture media. Each bar represents the mean \pm SD from three independent experiments. ${ }^{\star \star} P<0.01$ and ${ }^{* * *} P<0.001$ versus control; ${ }^{\# \#} P<0.01$ and ${ }^{\# \# \#} P<0.001$ versus aspirin treatment 
manner (Fig. 3A), indicating induction of autophagy. Autophagic flux, which was tracked using Ad-RFP-GFP-LC3, revealed that aspirin substantially enhanced the quantities of autophagosomes (yellow puncta) and autolysosomes (red puncta). This outcome was similar to the effects of rapamycin (Fig. 3B). Rapamycin, which is a pharmacological activator of autophagy, was used as a positive control. These data indicated that aspirin induces EC autophagy.

\section{Effects of Beclin-1 knockdown on aspirin-induced EC protection}

Beclin-1 primarily functions as a scaffolding protein to promote the formation of the Beclin-1/ Vps34 complex, which accelerates the autophagy cascade [18]. Therefore, we assessed the role of Beclin-1 in aspirin-induced HCAEC autophagy and protection. After Beclin-1 was successfully knocked down (Fig. 4A), aspirin treatment suppressed the increase in the LC3II/LC3I ratio and p-eNOS and NO levels but suppressed the decrease in p-p38, p-NF- $\kappa$ B, and p62 levels compared with those of NC (Fig. 4B and C). Moreover, sICAM-1 and sVCAM-1 levels increased after

A

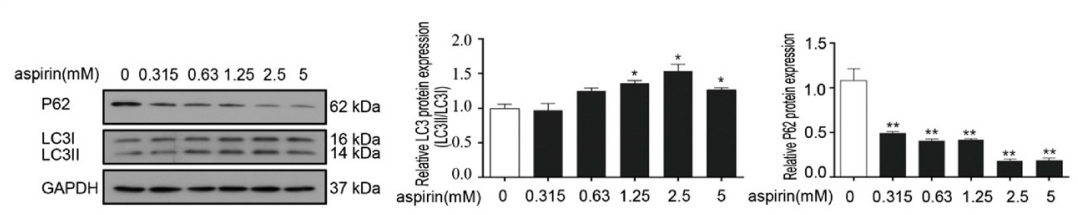

B
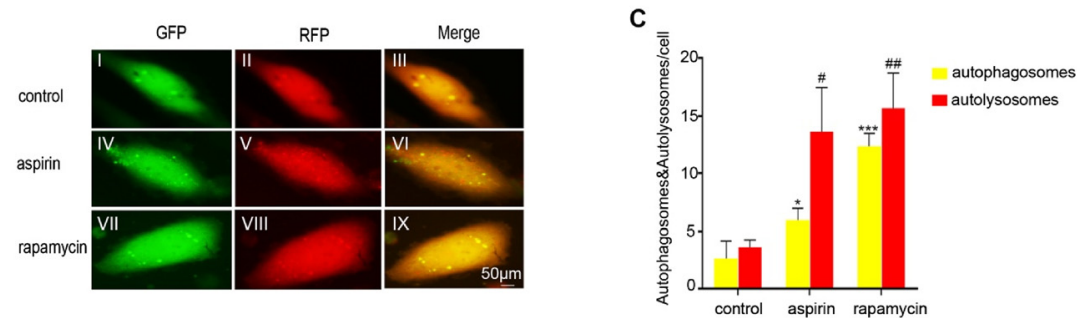

Fig. 3. Aspirin induced autophagy in the HCAECs. (A) HCAECs were incubated with aspirin at various concentrations $(0,0.315,0.63,1.25,2.5$, and $5 \mathrm{mM})$ for $16 \mathrm{~h}$. Cell lysates were subjected to immunoblotting assay with antibodies against LC3, p62, and GAPDH. The left panel shows the representative data. The right panel presents the band quantification of three experiments. Each bar represents the mean \pm SD calculated from three independent experiments. ${ }^{\star} P<0.05$ and ${ }^{* *} P<0.01$; aspirin treatment was compared with the control. (B) Autophagosomes and autolysosomes were tracked using mRFP-GFPLC3. HCAECs were infected with mRFP-GFP-LC3 at $50 \mathrm{MOI}$ for $24 \mathrm{~h}$, treated with or without aspirin (5 $\mathrm{mM})$ or rapamycin $(100 \mathrm{nM})$ for $16 \mathrm{~h}$, and analyzed via fluorescence microscopy. Autophagic flux was determined by counting autophagosomes (yellow puncta) and autolysosomes (red puncta) in the merged images. The green puncta represent GFP, the weakening of which can indicate the fusion of lysosomes and autophagosomes to form autolysosomes. (I) GFP of control. (II) RFP of control. (III) Merge of control. (IV) GFP of aspirin. (V) RFP of aspirin. (VI) Merge of aspirin. (VII) GFP of rapamycin. (VIII) RFP of rapamycin. (IX) Merge of rapamycin. Each bar represents the mean \pm SD calculated from three independent experiments. ${ }^{\star} P<0.05,{ }^{*} P<0.01$, and ${ }^{* * *} P<0.001$ versus control; ${ }^{\#} P<0.05$ and ${ }^{\#} P<0.01$ 
A

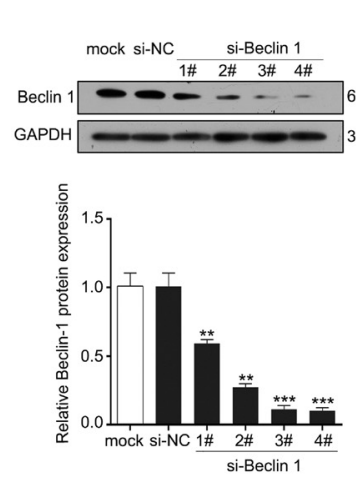

C

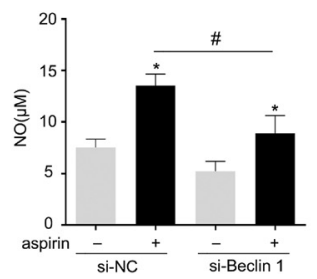

B

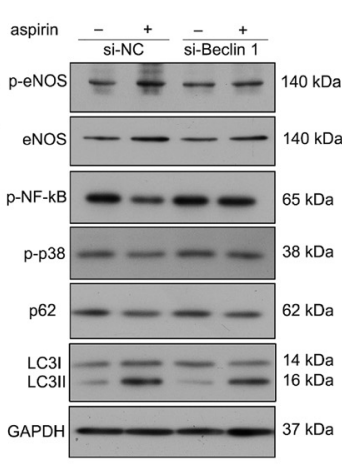

D

I

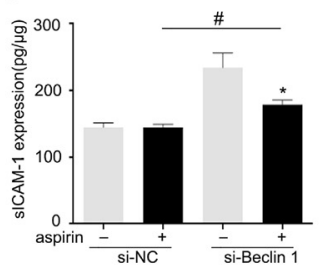

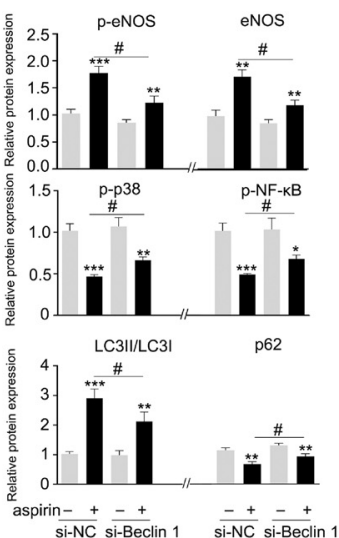

II

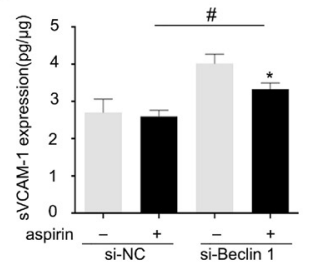

Fig. 4. Protective effects of aspirin were dependent on Beclin-1 in the HCAECs. (A) Beclin-1 protein expression was detected by immunoblotting assay in the HCAECs. Cells were transfected with $50 \mathrm{nM} \mathrm{NC}$ siRNA or $50 \mathrm{nM}$ Beclin-1 siRNA. (B) HCAECs were transfected with siRNA for $48 \mathrm{~h}$ and then treated with or without $2.5 \mathrm{mM}$ aspirin for an additional $16 \mathrm{~h}$. Cell lysates were subjected to immunoblotting assay with antibodies against LC3, p62, p-eNOS, eNOS, p38, and p-NF- $\kappa$ B or GAPDH. (C and D) Cell lysates were subjected to Griess reagent to measure NO levels and subjected to ELISA to determine (I) sICAM-1 and (II) sVCAM-1 expression levels. Experiments were replicated at least three times. Bars represent the mean $\pm \mathrm{SD} .{ }^{\star} P<0.05,{ }^{*} P<0.01$, and ${ }^{* * *} P<0.001$ : aspirin treatment versus control; ${ }^{\sharp} P<0.05$ : Beclin-1 siRNA versus NC siRNA. NC: negative control

Beclin-1 knockdown (Fig. 4D). These results suggested that aspirin-induced HCAEC autophagy and protection are dependent on Beclin-1.

\section{DISCUSSION}

The present study revealed that aspirin increased eNOS level and endogenous NO in the HCAECs under ox-LDL-, Ang-II-, and HG-induced injury by suppressing p38 and NF- $\kappa \mathrm{B}$ phosphorylation. Aspirin also decreased the expression levels of adhesion molecules (i.e., sICAM-1 and sVCAM-1). Beclin-1 was required for aspirin-induced autophagy to ameliorate endothelial dysfunction in the HCAECs. Therefore, aspirin protects HCAECs from ox-LDL-, Ang II-, and HG-induced injury in an autophagy-dependent manner, a 
process that increases eNOS expression and decreases the P38 MPAK and NF- $\kappa$ B signaling pathways (Fig. 5).

Vascular ECs can secrete various cytokines, such as NO, prostacyclin, endothelin-1, and von Willebrand factor, which are involved in regulating vascular tone, cell adhesion, smooth muscle cell proliferation, vascular inflammation and angiogenesis, and remodeling processes [1, 21]. As a signaling mediator, NO has diverse biological activities. Impaired NO bioavailability promotes endothelial dysfunction, a fundamental pathophysiological mechanism in the progression of cardiovascular and cerebrovascular diseases. Endothelial dysfunction participates in the development of various diseases, such as hypertension, atherosclerosis, coronary heart diseases, and diabetes. In the vasculature, NO is biosynthesized through eNOS catalytic activity. The results of the present study indicated that phosphorylated eNOS and total eNOS expression levels increased after treatment with aspirin in a dose-dependent manner. Decreased eNOS activity is the most important marker of EC dysfunction that results in impaired bioavailability of endogenous NO. Aspirin increased NO production in a dose-dependent manner. Evidence from explorative clinical trials suggests that treatment with low aspirin dose can help maintain vascular homeostasis by improving endothelium-dependent arterial relaxation [22]. The present study found that aspirin increased p-eNOS level and NO release in a dose-dependent manner, a result similar to that of a previous research [11].

Aspirin reduces the onset of cardiovascular diseases and inhibits inflammation and certain cancers. The NF- $\kappa \mathrm{B}$ and MAPK signaling pathways play important roles in the formation and development of atherosclerosis [23], hypertension [24], and diabetes [25, 26]. Ang-II can promote the formation of atherosclerosis by activating the NF- $\kappa \mathrm{B}$ and MAPK signal transduction pathways. Ang-II induces hypertensive endothelial injury in human umbilical vein ECs (HUVECs) via the Nrf2/NF- $\kappa \mathrm{B}$ pathways [24]. Animal studies have demonstrated that ox-LDL is involved in foam cell formation and aggravates atherosclerosis through the NF- $\kappa \mathrm{B}$ and p38 MAPK signal transduction pathways. Jayakumar et al. found that HG induces CAM expression and NF- $\kappa$ B activation in HUVECs [26]. In the present study, the HCAECs were pretreated with aspirin for $30 \mathrm{~min}$ and then cocultured with $50 \mu \mathrm{g} / \mathrm{mL}$ ox-LDL, $1 \mu \mathrm{M}$ Ang-II, and $25 \mathrm{mM} \mathrm{HG}$

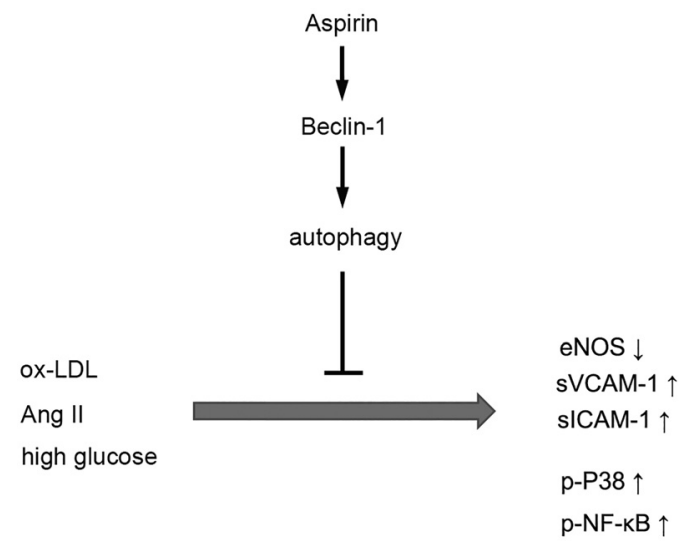

Fig. 5. Schematic of role of aspirin in the HCAECs. Aspirin protects HCAECs from ox-LDL-, Ang-II-, and HG-induced injury in an autophagy-dependent manner 
for $24 \mathrm{~h}$. Aspirin substantially inhibited the ox-LDL-, Ang II-, and HG-induced p38 and NF- $\kappa$ B activation in the HCAECs. Meanwhile, aspirin remarkably decreased the secretion of sICAM-1 and sVCAM-1, which were augmented by ox-LDL, Ang-II, and HG stimulation in the HCAECs. Results suggested that aspirin protects EC functions in ox-LDL-, Ang II-, and HG-induced cell injury.

Autophagy provides cellular metabolic precursors, especially in response to starvation, by degrading cellular components by lysosomes. Aspirin features an autophagic effect by activating the AMPK pathway and inhibiting mTOR signaling [27] or the p38/reactive oxygen species pathway in tumor cell lines [28] and heart fibrosis [29]. Nevertheless, the autophagic effects of aspirin on ECs have not been investigated yet. The LC3 II/I ratio was used as an indicator of autophagy-mitophagy flux. In autophagy, autophagosome degradation by lysosomes decreases SQSTM1/p62 levels. In the present study, aspirin increased the LC3II/LC3I ratio in the HCAECs in a dose-dependent manner. However, p62 expression gradually decreased, and the number of autophagosomes and autolysosomes substantially increased after $2.5 \mathrm{mM}$ aspirin treatment compared with the control. These data indicated that aspirin induces autophagy in ECs.

Beclin-1 plays an important role in the formation of autophagosomes and regulates autophagy [30]. In HUVECs, autophagy activation prevents cell death upon exposure to oxidative stress by activating Beclin-1 [31]. Beclin-1 knockdown inhibits autophagy and survival in oxLDL-treated HUVECs [32]. In the present study, Beclin-1 knockdown reduced the autophagic and endothelial protective effects of aspirin in the HCAECs. In addition, Beclin-1 knockdown in the HCAECs increased p38 MAPK phosphorylation and the NF- $\kappa$ B signaling pathway with aspirin treatment. Meanwhile, sICAM-1 and sVCAM-1 contents increased. Therefore, Beclin-1 is required for aspirin-induced autophagy to protect EC functions.

\section{CONCLUSIONS}

Aspirin can ameliorate EC dysfunction induced by ox-LDL, Ang-II, and HG by activating autophagy in a Beclin-1-dependent manner. The results provide additional experimental evidence and scientific rationale for aspirin treatment of cardiovascular diseases.

Conflict of interest: The authors declare no conflicts of interest.

\section{ACKNOWLEDGMENTS}

This study was supported by research grants from the National Natural Science Foundation of China (No. 81570259, 81873474, 81600350).

\section{REFERENCES}

1. Pearson JD. Normal endothelial cell function. Lupus 2000; 9: 183-8.

2. Rodríguez-Iturbe B, Johnson RJ. Heat shock proteins and cardiovascular disease. Physiol Int 2018; 105: 19-37. 
3. Qin W, Xie W, Xia N, He Q, Sun T. Silencing of transient receptor potential channel 4 alleviates oxLDLinduced angiogenesis in human coronary artery endothelial cells by inhibition of VEGF and NF-kappaB. Med Sci Monit 2016; 22: 930-6.

4. Muller DN, Heissmeyer V, Dechend R, Hampich F, Park JK, Fiebeler A, et al. Aspirin inhibits NF-kappaB and protects from angiotensin II-induced organ damage. FASEB J 2001; 15: 1822-4.

5. Fei Y, Sun L, Yuan C, Jiang M, Lou Q, Xu Y. CFTR ameliorates high glucose-induced oxidative stress and inflammation by mediating the NF-kappaB and MAPK signaling pathways in endothelial cells. Int J Mol Med 2018; 41: 3501-8.

6. Capodanno D, Angiolillo DJ. Aspirin for primary cardiovascular risk prevention and beyond in diabetes mellitus. Circulation 2016; 134: 1579-94.

7. Chang PY, Chen YJ, Chang FH, Lu J, Huang WH, Yang TC, et al. Aspirin protects human coronary artery endothelial cells against atherogenic electronegative LDL via an epigenetic mechanism: a novel cytoprotective role of aspirin in acute myocardial infarction. Cardiovasc Res 2013; 99: 137-45.

8. Furuno T, Yamasaki F, Yokoyama T, Sato K, Sato T, Doi Y, et al. Effects of various doses of aspirin on platelet activity and endothelial function. Heart Vessels 2011; 26: 267-73.

9. Ittaman SV, VanWormer JJ, Rezkalla SH. The role of aspirin in the prevention of cardiovascular disease. Clin Med Res 2014; 12: 147-54.

10. Ou HC, Lee WJ, Wu CM, Chen JF, Sheu WH. Aspirin prevents resistin-induced endothelial dysfunction by modulating AMPK, ROS, and Akt/eNOS signaling. J Vasc Surg 2012; 55: 1104-15.

11. Dragomir E, Manduteanu I, Voinea M, Costache G, Manea A, Simionescu M. Aspirin rectifies calcium homeostasis, decreases reactive oxygen species, and increases NO production in high glucose-exposed human endothelial cells. J Diabetes Complications 2004; 18: 289-99.

12. Mehta JL, Chen J, Yu F, Li DY. Aspirin inhibits ox-LDL-mediated LOX-1 expression and metalloproteinase-1 in human coronary endothelial cells. Cardiovasc Res 2004; 64: 243-9.

13. Maejima Y, Isobe M, Sadoshima J. Regulation of autophagy by Beclin 1 in the heart. J Mol Cell Cardiol 2016; 95: 19-25.

14. McCormick JJ, VanDusseldorp TA, Ulrich CG, Lanphere RL, Dokladny K, Mosely PL, et al. The effect of aging on the autophagic and heat shock response in human peripheral blood mononuclear cells. Physiol Int 2018; 105: 247-56.

15. Sun X, Du F, Liu S. Modulation of autophagy in exJSRV-env-transfected cells through the Akt/mTOR and MAPK signaling pathway. Biochem Biophys Res Commun 2017; 485: 672-8.

16. Kang R, Zeh HJ, Lotze MT, Tang D. The Beclin 1 network regulates autophagy and apoptosis. Cell Death Differ 2011; 18: 571-80.

17. Zhao Q, Wang Z, Wang Z, Wu L, Zhang W. Aspirin may inhibit angiogenesis and induce autophagy by inhibiting mTOR signaling pathway in murine hepatocarcinoma and sarcoma models. Oncol Lett 2016; 12: 2804-10.

18. Huang Z, Fang W, Liu W, Wang L, Liu B, Liu S, et al. Aspirin induces Beclin-1-dependent autophagy of human hepatocellular carcinoma cell. Eur J Pharmacol 2018; 823: 58-64.

19. He D, Zhao M, Wu C, Zhang W, Niu C, Yu B, et al. Apolipoprotein A-1 mimetic peptide 4F promotes endothelial repairing and compromises reendothelialization impaired by oxidized HDL through SR-B1. Redox Biol 2018; 15: 228-42.

20. Yang L, Cong HL, Wang SF, Liu T. AMP-activated protein kinase mediates the effects of lipoprotein-associated phospholipase A2 on endothelial dysfunction in atherosclerosis. Exp Ther Med 2017; 13: 1622-9.

21. Zhang S, Chen L, Zhou Z, Fan W, Liu S. Effects of Puerarin on clinical parameters, vascular endothelial function, and inflammatory factors in patients with coronary artery disease. Med Sci Monit 2019; 25: 402-8. 
22. Monobe H, Yamanari H, Nakamura K, Ohe T. Effects of low-dose aspirin on endothelial function in hypertensive patients. Clin Cardiol 2001; 24: 705-9.

23. Kardakaris R, Gareus R, Xanthoulea S, Pasparakis M. Endothelial and macrophage-specific deficiency of P38alpha MAPK does not affect the pathogenesis of atherosclerosis in ApoE-/- mice. PLoS One 2011; 6: e21055.

24. Chen J, Gong F, Chen MF, Li C, Hong P, Sun S, et al. In vitro vascular-protective effects of a tilapia byproduct oligopeptide on angiotensin II-induced hypertensive endothelial injury in HUVEC by Nrf2/NFkappaB pathways. Mar Drugs 2019; 17: 431.

25. Chu P, Han G, Ahsan A, Sun Z, Liu S, Zhang Z, et al. Phosphocreatine protects endothelial cells from Methylglyoxal induced oxidative stress and apoptosis via the regulation of PI3K/Akt/eNOS and NF-kappaB pathway. Vascul Pharmacol 2017; 91: 26-35.

26. Jayakumar T, Chang CC, Lin SL, Huang YK, Hu CM, Elizebeth AR, et al. Brazilin ameliorates high glucoseinduced vascular inflammation via inhibiting ROS and CAMs production in human umbilical vein endothelial cells. Biomed Res Int 2014; 2014: 403703.

27. Din FV, Valanciute A, Houde VP, Zibrova D, Green KA, Sakamoto K, et al. Aspirin inhibits mTOR signaling, activates AMP-activated protein kinase, and induces autophagy in colorectal cancer cells. Gastroenterology 2012; 142: 1504-15.e3.

28. Hernandez C, Barrachina MD, Vallecillo-Hernandez J, Alvarez A, Ortiz-Masia D, Cosin-Roger J, et al. Aspirin-induced gastrointestinal damage is associated with an inhibition of epithelial cell autophagy. J Gastroenterol 2016; 51: 691-701.

29. Liu PP, Liu HH, Sun SH, Shi XX, Yang WC, Su GH, et al. Aspirin alleviates cardiac fibrosis in mice by inhibiting autophagy. Acta Pharmacol Sin 2017; 38: 488-97.

30. Levine B, Kroemer G. Autophagy in the pathogenesis of disease. Cell 2008; 132: 27-42.

31. Xie Y, You SJ, Zhang YL, Han Q, Cao YJ, Xu XS, et al. Protective role of autophagy in AGE-induced early injury of human vascular endothelial cells. Mol Med Rep 2011; 4: 459-64.

32. Wang K, Yang C, Shi J, Gao T. Ox-LDL-induced lncRNA MALAT1 promotes autophagy in human umbilical vein endothelial cells by sponging miR-216a-5p and regulating Beclin-1 expression. Eur J Pharmacol 2019; 858: 172338 